\title{
UNA ORQUESTACIÓN INSTRUMENTAL DE LA SIMETRÍA AXIAL
}

\author{
AN INSTRUMENTAL ORCHESTRATION OF AXIAL SYMMETRY \\ UMA OROUESTRAÇÃO INSTRUMENTAL DA SIMETRIA AXIAL
}

\author{
Daysi Julissa García-Cuéllar \\ Pontificia Universidad Católica del Perú, Lima, Perú \\ Recibido: 01/05/2021 - Aceptado: 15/06/2021 - Publicado: 01/12/2021 \\ Remita cualquier duda sobre esta obra a: Daysi Julissa García-Cuéllar. \\ Email: garcia.daysi@pucp.pe
}

\begin{abstract}
RESUMEN
El presente artículo muestra un estudio de corte cualitativo que tiene como objetivo describir cómo organizar el trabajo de aula para propiciar el aprendizaje de la simetría axial mediado con GeoGebra. Para ello, se basa como marco teórico en la Aproximación Instrumental y a la Orquestación Instrumental. La parte experimental fue realizada con una docente-investigadora y treinta y seis estudiantes de primero de secundaria de una institución educativa privada de Lima - Perú. Los resultados muestran que las fases de la orquestación instrumental permiten tomar decisiones previas, es decir, la configuración didáctica la cual permite elegir, según las herramientas y las restricciones institucionales, las actividades a realizar y los artefactos a implementar. La fase de desempeño didáctico permite reconocer las decisiones ad hoc lo cual posibilita implementar nuevas organizaciones con el fin de cumplir con los objetivos de aprendizaje. GeoGebra permitió a los estudiantes conjeturar, deducir y comprobar sus ideas para generar esquemas de acción colectiva instrumentada.
\end{abstract}

Palabras clave: Génesis instrumental; Orquestación Instrumental; Instrumento; GeoGebra.

\begin{abstract}
This article shows a qualitative study that aims to describe how to organize classroom work to promote the learning of axial symmetry mediated with GeoGebra. For this, it is based as a theoretical framework on the Instrumental Approach and Instrumental Orchestration. The experimental part was carried out with a teacher-researcher and thirty-six first year high school students from a private educational institution in Lima - Peru. The results show that the phases of the instrumental orchestration allow making prior decisions, that is, the didactic configuration which allows choosing, according to the tools and institutional restrictions, the activities to be carried out and the artifacts to be implemented. The didactic performance phase allows the recognition of ad hoc decisions which makes it possible to implement new organizations in order to meet the learning objectives. GeoGebra allowed students to conjecture, deduce, and test their ideas to generate instrumented collective action schemes.
\end{abstract}

Keywords: Instrumental genesis; Instrumental orchestration; Instrument; GeoGebra. 


\section{RESUMO}

Este artigo apresenta um estudo qualitativo que objetiva descrever como organizar o trabalho em sala de aula para promover a aprendizagem da simetria axial mediada pelo GeoGebra. Para tanto, baseia-se como referencial teórico na Abordagem Instrumental e Orquestração Instrumental. A parte experimental foi realizada com uma professora-pesquisadora e trinta e seis alunos do primeiro ano do ensino médio de uma instituição de ensino privada de Lima - Peru. Os resultados mostram que as fases da orquestração instrumental permitem a tomada de decisóes prévias, ou seja, a configuração didática que permite escolher, de acordo com as ferramentas e restrições institucionais, as atividades a serem realizadas e os artefatos a serem implementados. A fase de desempenho didático permite o reconhecimento de decisões ad hoc que possibilitam a implantação de novas formas de organizações para cumprir os objetivos de aprendizagem. O GeoGebra permitiu que os alunos conjeturassem, deduzissem e testassem suas ideias para gerar esquemas de ação coletiva instrumentadas.

Palavras-chave: Gênese instrumental; Orquestração instrumental; Instrumento; GeoGebra.

\section{INTRODUCCIÓN}

En el actual currículo peruano el contenido de simetría axial se encuentra en los ciclos $V$ que corresponde al quinto y sexto grado de educación primaria ( 10 y 11 años de edad) y al séptimo ciclo, que corresponden al primer y segundo grado de educación secundaria (12 y 13 años de edad). Este objeto matemático se encuentra dentro de la competencia denominada resuelve problemas de regularidad, equivalencia y cambio que concentra temas de Geometría.

Los resultados de la Evaluación Censal de Estudiantes (ECE), aplicada a los alumnos de $2^{\circ}$ grado de Educación Secundaria matriculados oficialmente en el sistema educativo peruano en el año 2015 y en el 2016, mostrados en la Figura 1, indican que del 2015 al 2016 hubo una pequeña mejora en los resultados en los aprendizajes en el área de la matemática. Sin embargo, en el 2016, que fue la última aplicación de una ECE, solo el 11,5\% de los estudiantes del grado mencionado, se encontraron en el nivel satisfactorio.

\section{Figura 1}

Resultados de la Evaluación Censal de Estudiantes 2015-2016(Perú, 2016)

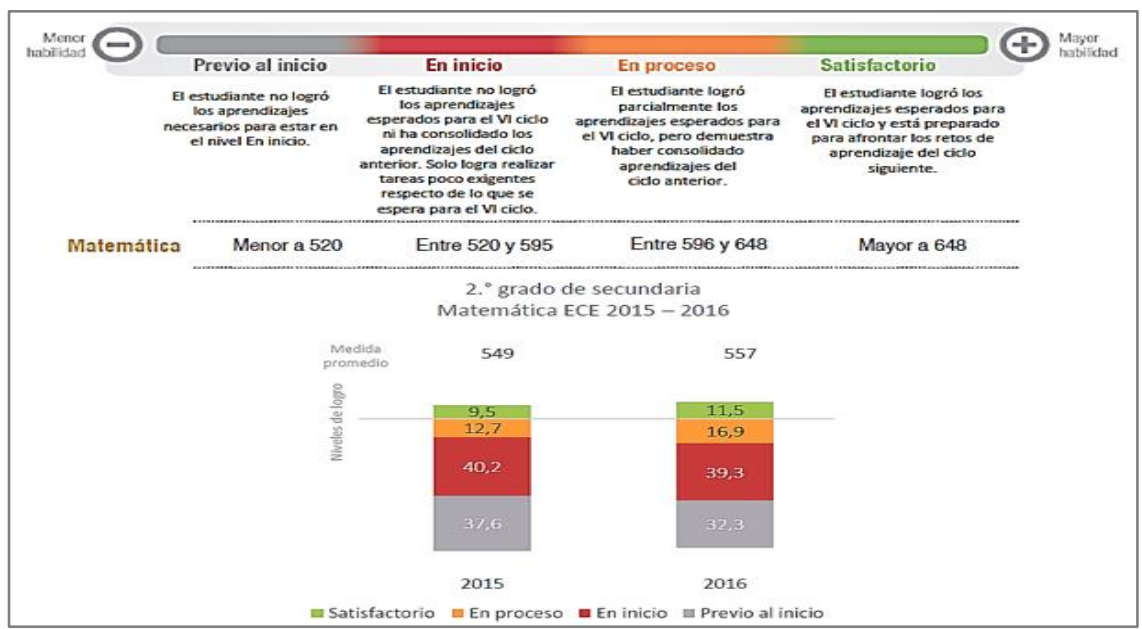


De estos resultados en las pruebas aplicadas a los estudiantes, podemos apreciar que éstos todavía tienen dificultades en el aprendizaje de la matemática y en especial de la Geometría. Según García-Cuéllar (2014), hay temas como las transformaciones geométricas en el plano, la geometría analítica, entre otros; que no se enseñan a pesar de que están en el currículo, pensamos que esto sucede por falta de tiempo o porque se profundiza otros temas, en especial el cálculo aritmético o el cálculo algebraico, es decir, aprendizaje de algoritmos.

Dentro de las transformaciones geométricas en el plano se encuentra la simetría, ésta tiene varias aplicaciones como lo indica Lederman:

[...] la simetria impregna toda la ciencia y ocupa un lugarprominente en la quimica, la biología, la fisiología y la astronomía. La simetría abarca desde el mundo intimo de la estructura de la materia hasta el inmenso dominio cósmico, pasando por el universo abstracto de las matemáticas. (Lederman, 1996, p. 229).

Según García-Cuéllar (2014), la simetría es un contenido matemático importante debido a que tiene implicancia en el aprendizaje de otros contenidos o conocimientos que los estudiantes van a aprender en los últimos años de educación secundaria y/o primeros ciclos universitarios como, por ejemplo: el aprendizaje de la parábola, elipse e hipérbola, así como también en el aprendizaje de las funciones inversas y funciones pares. La simetría proporciona oportunidades para que los estudiantes visualicen la geometría en el mundo desde el arte, la naturaleza, las construcciones arquitectónicas, como ya lo hemos mencionado anteriormente, pero lo más importante es que permite la exploración de regularidades y/o patrones que representan la esencia del conocimiento matemático.

En Educación Matemática existen diversos estudios, como los realizados por Ferreira (2005), Jaime (1993), Lima (2006) y Ulian (2008), los cuales muestran que existe una problemática en la enseñanza y en el aprendizaje de la simetría axial, como el de identificación de figuras simétricas y problemas de construcción de figuras simétricas. Otra investigación como la de Ferreira (2005) que realizó un estudio de las isometrías dando énfasis a la simetría, donde hace un análisis en los documentos curriculares como la Propuesta Curricular Nacional del Brasil y también analiza las actividades de algunos textos escolares presentan nociones de isometrías y con ello el estudio de la simetría. La investigadora manifiesta que no hay un estudio profundo de la noción de simetría en los textos escolares, por ello, menciona que la simetría es uno de los contenidos que no se profundiza en los libros de textos escolares del nivel medio en Brasil y que es necesario realizar más estudios. Este problema es similar en el Perú, en los libros didácticos el objeto matemático Simetría axial es presentado como acabado, es decir, solo necesita ser aplicado por los estudiantes, como una repetición de modelos dados en los ejercicios resueltos o ejemplos. 


\section{Figura 2}

Libro de actividades Matemática 1 (Santillana, 2017, p. 183)
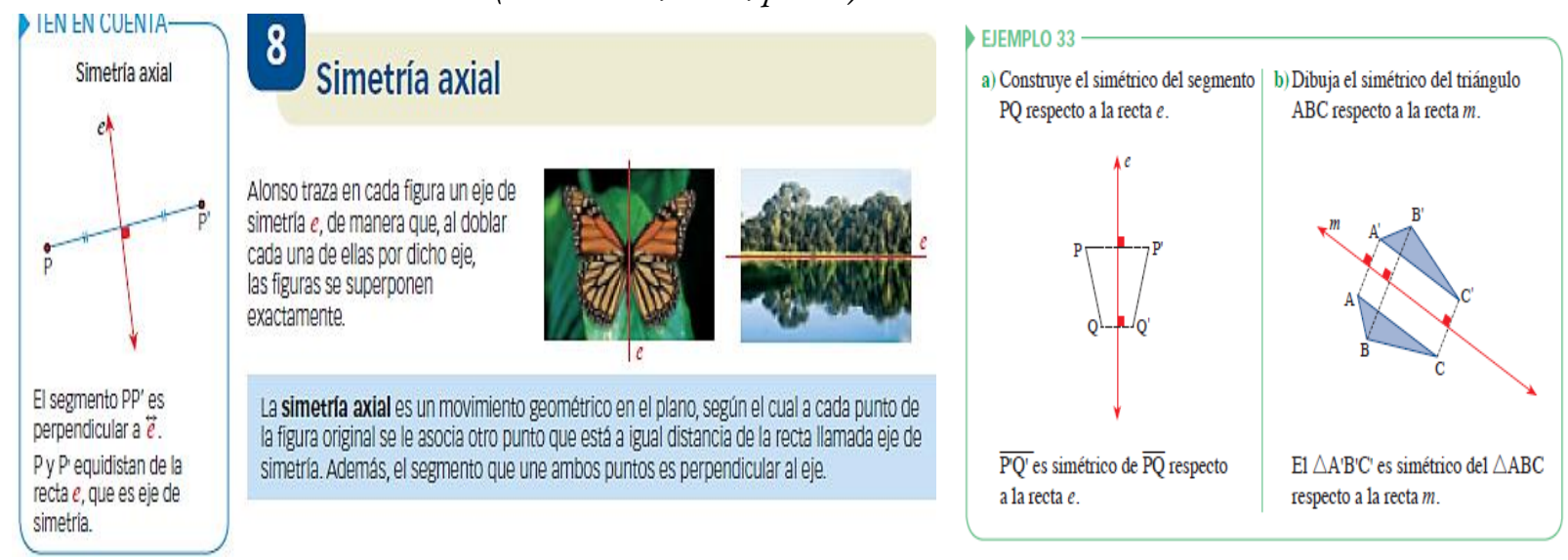

Como se puede apreciar, en la Figura 2 se muestra cómo es introducido el objeto matemático Simetría axial, en un libro de actividades de Matemática 1 del primer grado de Educación Secundaria en Perú. También se puede observar que el concepto de simetría axial es presentado sin actividades que permitan a los estudiantes deducir, analizar, verificar y descubrir este concepto. Luego, son presentados ejemplos de ejercicios propuestos donde se muestra cómo trazar el simétrico de una figura o cómo determinar el eje de simetría. Posteriormente, las actividades propuestas son del mismo tipo que los ejemplos, es decir, el estudiante solo repite los modelos de solución dados en el libro.

Por otro lado, Gallegos y Peña (2012) indican que en las últimas décadas, muchas pesquisas en el área de Educación Matemáticas se centran en el uso de tecnologías para la enseñanza y el aprendizaje de contenidos matemáticos. Precisamente, ellos afirman que:

La integración y utilización de las tecnologias en el proceso educativo de matemáticas es un asunto que viene ocupando el trabajo de los investigadores en educación matemática. Las investigaciones tratan de determinar los posibles beneficios que la utilización de las tecnologías conlleva, asi como diversas metodologias y entornos interactivos multimedia de aprendizaje que produzcan mejoras en los procesos de enseñanza y aprendizaje. (Gallegos \& Peña, 2012, p. 11)

Además de esta investigación, el Ministerio de Educación del Perú en sus Orientaciones para el trabajo pedagógico también menciona la importancia y pertinencia del uso de la tecnología, especialmente, en Geometría.

[...] La tecnología desempeña también un papel importante en la enseñanza y el aprendizaje de la geometria. Herramientas como un programa informático de "Geometria dinámica", capacitan 
para modelizar una gran variedad de figuras de dos dimensiones y para tener una experiencia interactiva con ellas. La visualización y el razonamiento espacial se enriquecen mediante la interacción con animaciones de ordenador y en otros contextos tecnológicos. (Perú, 2011, p. 30)

Por otro lado, el Proyecto Educativo Nacional (PEN) al 2021 (Perú, 2007) propone estructurar y fortalecer la formación de profesores de matemática. En el documento oficial, el tercer objetivo estratégico señala que un profesor de matemática debe estar preparado para proponer y desarrollar problemas para que los estudiantes sean capaces de resolverlos, establecer relaciones entre objetos matemáticos, etc. Sin embargo, en la evaluación a profesores que ejercen la carrera pública magisterial realizada el 2015, de un total de 19239 docentes evaluados, el 47,7\% lograron el límite inferior, en consecuencia, no alcanza el nivel mínimo de desempeño docente. Estas evidencias, confirman la necesidad de contribuir con la formación de profesores de matemática en relación al dominio de la Geometría.

Por todo lo mencionado anteriormente, en este artículo se presenta una Orquestación Instrumental de la simetría axial, es decir, cómo el docente organiza y lleva al aula actividades cuyo objeto matemático es la simetría axial y que son mediadas por tecnología, específicamente por GeoGebra para generar las Génesis Instrumental de sus estudiantes. A partir del estudio realizado por García-Cuéllar (2014), que tuvo como objetivo analizar el proceso de Génesis Instrumental de la simetría axial mediada por GeoGebra en estudiantes de primer grado de secundaria (12 o 13 años), este estudio continúa con la investigación y se centra en las acciones del profesor para orquestar las génesis instrumentales de los estudiantes. Por ello, usamos como marco teórico al Enfoque instrumental de Rabardel (1995) y a la Orquestación Instrumental de Trouche y Drijvers (2014), que se explica a continuación.

\section{ASPECTOS TEÓRICOS}

En este estudio cualitativo se utilizó como referencial teórico a la Aproximación Instrumental y la Orquestación Instrumental, las cuales describiremos a seguir.

\section{APROXIMACIÓN INSTRUMENTAL}

El Enfoque Instrumental aborda la dimensión tecnológica de la educación matemática, articulando los aspectos importantes de su integración en el proceso de enseñanza y aprendizaje de las matemáticas.

De acuerdo a Rabardel (1995), el Enfoque Instrumental estudia la diferencia que existe entre el artefacto, instrumento y los procesos que desenvuelven la transformación progresiva del artefacto en instrumento, transformación que denominó como proceso Génesis Instrumental. El autor considera tres polos importantes en la Génesis instrumental, estos son: el sujeto, que puede ser un usuario, operario, trabajador o agente; el instrumento, que se refiere a la herramienta, máquinas, sistemas, 
utensilio, etc.; y el objeto, al cual va dirigida la acción con ayuda del instrumento, este puede ser la materia prima, objeto de la actividad o trabajo.

El investigador sostiene que el instrumento no existe en sí, sino que es el resultado de asociar el artefacto a la acción del sujeto, como medio para la misma. El autor señala que el artefacto pasará al estado de instrumento, cuando el sujeto le asigne los esquemas de utilización correspondientes.

En cuanto a la génesis instrumental, esta consta de dos dimensiones: La instrumentalización y la instrumentación como se puede observar en la Figura 3.

\section{Figura 3}

Proceso de la Génesis Instrumental (adaptado de Trouche, 2004)

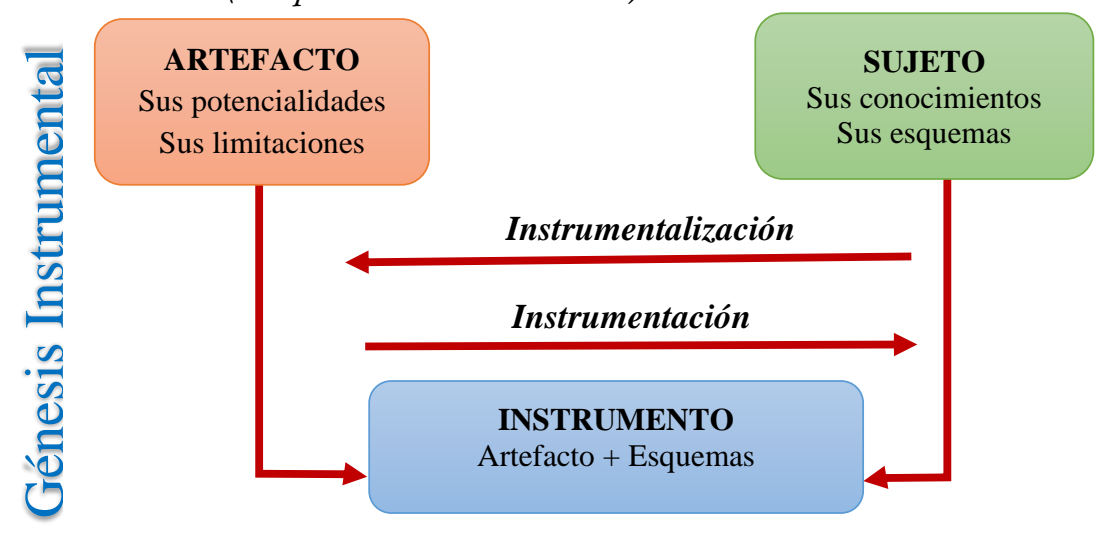

Los procesos de instrumentalización están dirigidos hacia el artefacto: selección, agrupación, producción e institución de funciones, usos desviados, atribuciones de propiedades, transformaciones del artefacto, de su estructura, de su funcionamiento, etc. [...] los procesos de Instrumentación están relacionados con el sujeto: con la emergencia y evolución de los esquemas sociales de utilización y de acción instrumentada: su constitución, su evolución por acomodación, coordinación y asimilación reciproca, la asimilación de artefactos nuevos a los esquemas ya constituidos, etc. (Rabardel, 1995, p. 215)

En resumen, las dos dimensiones de la Génesis Instrumental, dependen de su orientación: La instrumentalización está dirigida hacia la parte artefactual del instrumento, consta del enriquecimiento de las propiedades del artefacto por parte del sujeto. Es decir, es el resultado de la atribución de una función al artefacto por parte del sujeto. La instrumentación está dirigida hacia el sujeto. Se refiere a la construcción de esquemas de uso por parte del sujeto, relativos a la ejecución de ciertas tareas. En este proceso se lleva a cabo la asimilación de nuevos artefactos a los esquemas y la acomodación de los esquemas para dar nuevos significados a los artefactos. 
Rabardel (2011), a partir de esta noción de esquema de Vergnaud (1996), define los esquemas de utilización como el conjunto estructurado de las características generalizables de la acción que permiten repetir la misma acción o aplicarlas en nuevos contextos. Estos esquemas, a la vez, pueden ser clasificados en tres estatus, ver Figura 4, en esquemas de uso (dirigidas a tareas secundarias), esquemas de acción instrumentada (dirigidas a la tarea principal o primaria) y esquemas de acción colectiva instrumentada (cuando el colectivo comparte el mismo instrumento o trabaja con la misma clase de instrumento, buscando alcanzar una meta en común).

\section{Figura 4}

Estatus de los esquemas de utilización (adaptado de Rabardel, 2011)

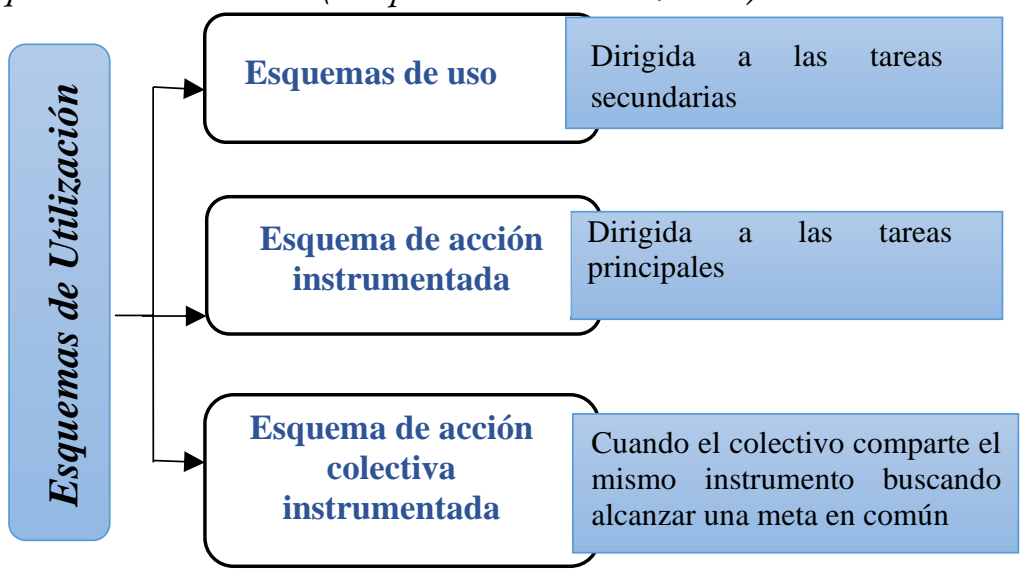

\section{ORQUESTACIÓN INSTRUMENTAL}

La Orquestación Instrumental surge a partir de reconocer la importancia de la función de las tareas o situaciones para generar el proceso de la Génesis Instrumental, y el papel fundamental del profesor, quien es el que propone dichas tareas para, justamente, buscar el desarrollo de las génesis instrumentales en sus estudiantes. Es por ello que los términos de artefacto, instrumento, esquemas de utilización, instrumentalización, instrumentación y génesis instrumental, tienen el mismo sentido que en la Aproximación Instrumental.

Trouche (2004) utiliza la noción de Orquestación de manera metafórica con el propósito de describir la gestión que hace el profesor de los instrumentos individuales en los procesos de aprendizaje colectivo, en el sentido de que las génesis instrumentales necesitan ser monitoreadas por el profesor a través de la orquestación de situaciones matemáticas.

Según Trouche, una Orquestación Instrumental (OI) es el arreglo sistemático e intencional de los elementos (artefactos y seres humanos) de un ambiente, realizado por un agente (profesor) con el fin de hacer efectiva una situación dada y, en general, guiar a los aprendices en las génesis instrumentales y en la evolución y equilibrio de sus sistemas de instrumentos. En la Figura 5, se muestran los componentes de una OI. 


\section{Figura 5}

Componentes de una OI (adaptado de Bellemain E' Trouche, 2016)

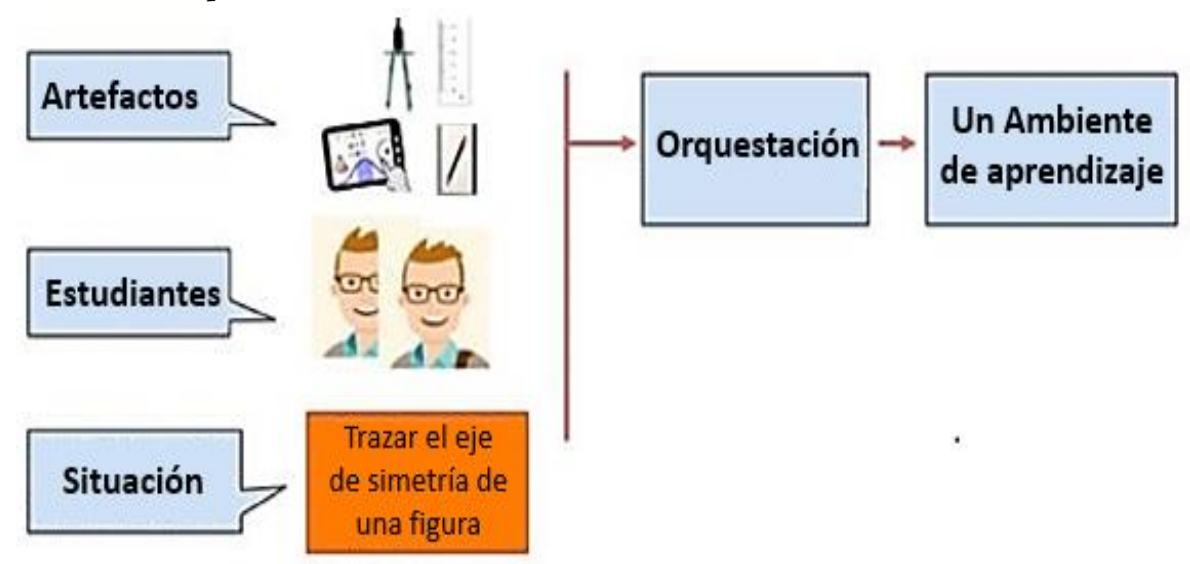

Según Trouche (2004), la Orquestación Instrumental involucra dos fases: Configuración didáctica y Modo de ejecución. Al respecto, Drijvers (2012) adiciona una tercera fase que se denomina Desempeño didáctico.

La fase de configuración didáctica se refiere al arreglo de los componentes en el ambiente de aprendizaje, es decir, la configuración del entorno en donde se llevará a cabo los procesos de enseñanza y aprendizaje, así como los artefactos que participarán en ella. El modo de ejecución alude a la forma cómo el docente decide ejecutar las acciones que permitan lograr la intensión sobre una tarea determinada. Finalmente, el desempeño didáctico concierne a las acciones realizadas, tanto por los estudiantes y como por el docente, frente a una situación matemática propuesta; es decir, es la toma de decisiones frente a algunas situaciones que permitan garantizar el propósito de la tarea o actividad.

Tabach (2013, citado en Şay \& Akkoç, 2015) distingue 10 tipos de Orquestación, las cuales se presentan en la Tabla 1 que sigue a continuación. 


\section{Tabla 1}

Tipos de Orquestación Instrumental (Traducido de Tabach, 2013, citado en Şay E Akkog, 2015)

\begin{tabular}{|c|c|c|}
\hline Tipo de orquestación & Configuración didáctica & Modo de ejecución \\
\hline $\begin{array}{l}\text { Demostración técnica. } \\
\text { (Drijvers et al., 2010) }\end{array}$ & $\begin{array}{l}\text { Clase completa entorno a } \\
\text { una pantalla central }\end{array}$ & $\begin{array}{l}\text { El docente explica los detalles técnicos para usar la } \\
\text { herramienta }\end{array}$ \\
\hline $\begin{array}{l}\text { Explicar la pantalla. } \\
\text { (Drijvers et al., 2010) }\end{array}$ & $\begin{array}{l}\text { Clase completa entorno a } \\
\text { una pantalla central }\end{array}$ & $\begin{array}{l}\text { Las explicaciones del docente van más allá de las } \\
\text { técnicas e involucran contenidos matemáticos. }\end{array}$ \\
\hline $\begin{array}{l}\text { Vincular el tablero de la } \\
\text { pantalla (Drijvers et al., } \\
\text { 2010) }\end{array}$ & $\begin{array}{l}\text { Clase completa entorno a } \\
\text { una pantalla central }\end{array}$ & $\begin{array}{l}\text { El docente conecta en la pantalla representaciones de } \\
\text { los mismos objetos matemáticos que aparecen en el } \\
\text { libro o en la pizarra. }\end{array}$ \\
\hline $\begin{array}{l}\text { Sherpa en el trabajo } \\
\text { (Drijvers et al., 2010) }\end{array}$ & $\begin{array}{l}\text { Clase completa entorno a } \\
\text { una pantalla central }\end{array}$ & $\begin{array}{l}\text { La tecnología está en manos de un estudiante, que } \\
\text { la lleva a toda la clase para su discusión. }\end{array}$ \\
\hline $\begin{array}{l}\text { Tecnología sin uso. } \\
\text { (Tabach, 2013) }\end{array}$ & $\begin{array}{l}\text { Clase completa entorno a } \\
\text { una pantalla central }\end{array}$ & $\begin{array}{l}\text { La tecnología está disponible pero el docente decide } \\
\text { no usarla. }\end{array}$ \\
\hline $\begin{array}{l}\text { Discutir la pantalla. } \\
\text { (Drijvers et al., 2010) }\end{array}$ & $\begin{array}{l}\text { Clase completa entorno a } \\
\text { una pantalla central }\end{array}$ & $\begin{array}{l}\text { Discusión de toda la clase guiada por el docente para } \\
\text { potenciar la génesis instrumental colectiva. }\end{array}$ \\
\hline $\begin{array}{c}\text { Parar y mostrar } \\
\text { (Drijvers et al., 2010) }\end{array}$ & $\begin{array}{l}\text { Clase completa entorno a } \\
\text { una pantalla central }\end{array}$ & $\begin{array}{l}\text { El docente trae a colación el trabajo anterior de un } \\
\text { estudiante que había almacenado e identificado } \\
\text { como relevante para su posterior discusión. }\end{array}$ \\
\hline $\begin{array}{l}\text { Trabajar y caminar. } \\
\text { (Drijvers, 2012) }\end{array}$ & $\begin{array}{l}\text { Los estudiantes trabajan } \\
\text { individualmente o en pares } \\
\text { con computadoras }\end{array}$ & $\begin{array}{l}\text { El docente camina entre los estudiantes, } \\
\text { monitoreando su progreso y proporciona } \\
\text { orientación cuando surge la necesidad }\end{array}$ \\
\hline $\begin{array}{l}\text { Discutir la tecnología } \\
\text { sin ella (Tabach, 2013) }\end{array}$ & $\begin{array}{l}\text { Cada estudiante tiene una } \\
\text { laptop o usa computadoras } \\
\text { que traen al salón con } \\
\text { vehículos con ruedas. }\end{array}$ & $\begin{array}{l}\text { El docente usa el sistema de transporte móvil } \\
\text { necesita computadoras en la enseñanza. }\end{array}$ \\
\hline $\begin{array}{l}\text { Monitorear y guiar } \\
\text { (Tabach, 2011) }\end{array}$ & & $\begin{array}{l}\text { El docente utiliza un sistema de gestión del } \\
\text { aprendizaje para orientar a los estudiantes. }\end{array}$ \\
\hline
\end{tabular}

A continuación, se presenta la metodología y sujetos de investigación.

\section{METODOLOGÍA DE LA INVESTIGACIÓN}

La metodología de esta investigación es cualitativa, de naturaleza descriptiva e interpretativa. Según Bogdan y Biklen (2007), la investigación cualitativa en el campo de la educación tiene las siguientes características: (i) el ambiente natural es la fuente directa de los datos, (ii) los resultados tienen un fuerte componente descriptivo, (iii) los investigadores se preocupan más en los procesos que en los productos, (iv) el análisis de los datos es generalmente intuitivo, y (v) el significado es de esencial importancia en este enfoque.

El estudio se desarrolló con una docente y 36 estudiantes de 12 o13 años de edad de primer año de secundaria de una institución educativa privada de Lima-Perú, en un aula de informática con 
computadoras, una por estudiante. Asimismo, en el aula se tenía un proyector de pantalla y las computadoras de los estudiantes se interconectaban bajo una red interna con el computador de la docente.

\section{LA EXPERIENCIA}

Dado que la Orquestación Instrumental busca entender y modelar la acción del docente, tomando por base tres fases: La configuración didáctica, el modo de operación y el desempeño didáctico, caracterizadas por Trouche y Drijvers (2014). A seguir se describe cada una de las fases que han sido consideradas por una docente de matemática para configurar una Orquestación Instrumental de la Simetría axial para estudiantes de primer grado de secundaria en Perú.

\section{FASE DE CONFIGURACIÓN DIDÁCTICA}

En la fase de configuración didáctica, el ambiente de aprendizaje se dio en la sala de informática que contaba con 38 computadoras. Los artefactos usados fueron GeoGebra, proyector de pantalla, lápiz, regla y papel. Los sujetos fueron una docente-investigadora y 36 estudiantes de primero de secundaria entre 12 y 13 años de edad. Se realizaron 3 encuentros. Las situaciones a desarrollar propuestas por García-Cuéllar (2014), fueron de tres tipos: la actividad $N^{\circ} 0$, que tuvo la finalidad de familiarizar a los estudiantes con algunas herramientas del GeoGebra; la actividad $\mathrm{N}^{\circ} 1$, que se centró en el desarrollo de esquemas de utilización de la simetría axial y, finalmente, la actividad $\mathrm{N}^{\circ} 2$, donde se buscó que los estudiantes pongan en acción sus esquemas de utilización. Cabe mencionar que las actividades pueden ser observadas en https://www.geogebra.org/m/w4e6sapz. Esta organización se puede observar en la Tabla 2 que se presenta a continuación.

\section{Tabla 2}

Descripción de los encuentros de aplicación de las actividades (Garcia-Cuéllar, 2014)

\begin{tabular}{lccc}
\hline \multicolumn{1}{c}{ Actividad } & Encuentro & Contenido & Tipo de O. I \\
\hline Actividad $\mathrm{N}^{\circ} 0$ & I & Introducción al GeoGebra & Sherpa en el trabajo \\
\hline Actividad $\mathrm{N}^{\circ} 1(\mathrm{~A}, \mathrm{~B}, \mathrm{C}, \mathrm{D}$ y E) & I y II & Simetría Axial & $\begin{array}{c}\text { Trabajar y caminar } \\
\text { Discutir la pantalla. }\end{array}$ \\
\hline Actividad $\mathrm{N}^{\circ} 2(\mathrm{~A}, \mathrm{~B}$ y C $)$ & II & Aplicaciones & $\begin{array}{c}\text { Trabajar y caminar } \\
\text { Sherpa en el trabajo }\end{array}$ \\
\hline
\end{tabular}

En cuanto al tipo de orquestación propuesto por Tabach (2013, citado en Şay \& Akkoç, 2015), en la actividad $\mathrm{N}^{\circ} 0$ fue utilizado sherpa en el trabajo en donde se usó el proyector (pantalla central) para explicar el uso de las herramientas del GeoGebra que eran necesarias conocer para resolver las actividades propuestas (instrumentalización de las herramientas del GeoGebra) y luego, en la actividad $N^{\circ} 1$ fue usado trabajar y caminar en donde los estudiantes trabajan individualmente o en pares con el uso de 
computadoras y la docente camina entre los estudiantes, monitoreando su progreso y proporciona orientación cuando surge la necesidad y luego, fue usado discutir en la pantalla, en donde la discusión de toda la clase, guiada por la docente, buscó potenciar la génesis instrumental colectiva a partir de los esquemas de utilización de los estudiantes. Para la actividad $\mathrm{N}^{\circ} 2$, fue utilizado en un primer momento trabajar y caminar y posteriormente, sherpa en el trabajo, ya explicados.

En la Figura 6, se puede observar los tipos de Orquestación utilizados en la experiencia por la docente-investigadora para generar las génesis instrumentales de la simetría axial mediada por el GeoGebra en sus estudiantes.

\section{Figura 6}

Tipos de orquestación utilizadas en la experiencia (datos de la investigación - Garcia-Cuéllar, 2014)

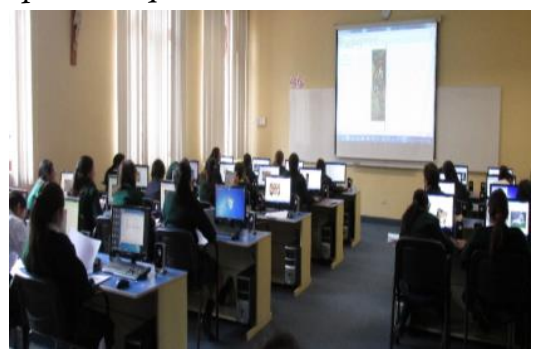

Sherpa en el trabajo

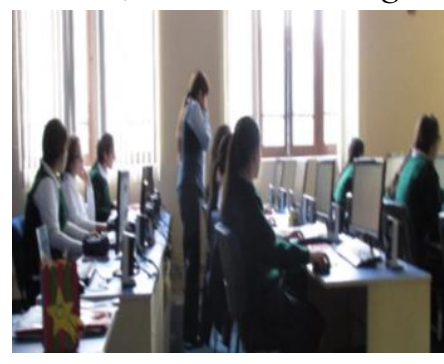

Trabajar y caminar

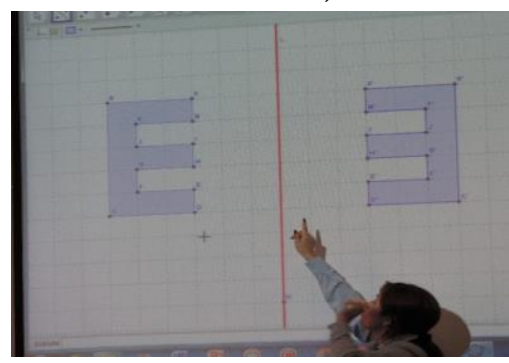

Discutir en la pantalla

A manera de resumen, en la Figura 7 se presenta los aspectos elegidos en la fase de configuración didáctica en cuanto al ambiente, los artefactos, los sujetos, el tema u objeto matemático de estudio y las actividades a realizar en la experiencia.

\section{Figura 7}

Esquema de los aspectos elegidos en la fase configuración didáctica

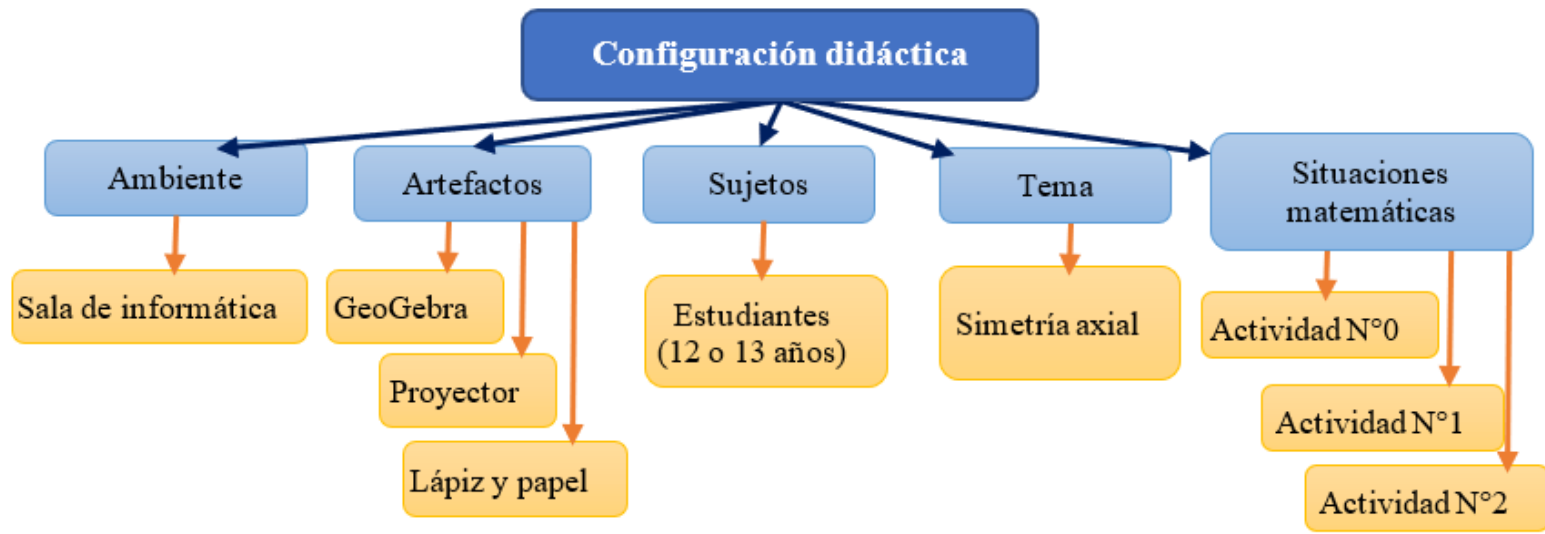




\section{FASE MODO DE EXPLORACIÓN}

En la fase modo de exploración, basados en el estudio de Couto (2015), se dividió en tres relaciones: la pedagógica, la organizacional y la didáctica.

En cuanto a la relación pedagógica, se consideraron las orientaciones a los estudiantes para que sean actores principales de la resolución de las actividades, las actividades no fueron evaluadas si no que estuvieron enmarcadas de su proceso formativo.

En la relación organizacional, los estudiantes desarrollaron las actividades de manera individual y luego, de un tiempo dado por la docente se realizaba un proceso de socialización de las respuestas por medio del uso del proyector de pantalla. También la docente caminaba entre los estudiantes, monitoreando su progreso y proporcionaba orientación cuando surgía la necesidad.

En la relación didáctica, se observó que GeoGebra permitió que los estudiantes realizaran las actividades de manera sencilla dado que se familiarizaron rápidamente con las herramientas de este software de Geometría dinámica. Las estrategias utilizadas de trabajo individual y luego grupal permitieron compartir manera de solución y generar esquemas de acción colectivas instrumentadas dado que todo el grupo participaba.

A seguir se presenta tres actividades que son la actividad $1 \mathrm{C}$, la actividad $2 \mathrm{~A}$ y la actividad 2C, estas actividades son las propuestas en García-Cuéllar (2014) y presentadas también en García-Cuéllar y Salazar (2019).

\section{ACTIVIDAD $1 \mathrm{C}$}

A seguir se presenta el enunciado y el análisis didáctico de la actividad 1C.

\section{Figura 8}

\section{Enunciado de la Actividad 1C (Garcia-Cuéllar, 2014)}

Abre el archivo actividad1_C.ggb.Utilizando la herramienta punto
medio, traza los puntos medios de los segmentos AA', BB', CC',
DD', EE', FF', GG', HH' e II'. Con la herramienta recta, traza la recta
que pasa por los puntos medios marcados anteriormente. Anota tus
observaciones.
Traza el segmento AA' y mide un ángulo que se forma entre la
intersección del segmento y la recta. ¿Cuánto mide el ángulo?
Traza BB' y mide un ángulo que se forma entre la intersección del
segmento y la recta. ¿Cuánto mide el ángulo? ¿Qué puedes concluir
con respectos a los ángulos que se forman entre la intersección de la
recta y los segmentos trazados? Justifica

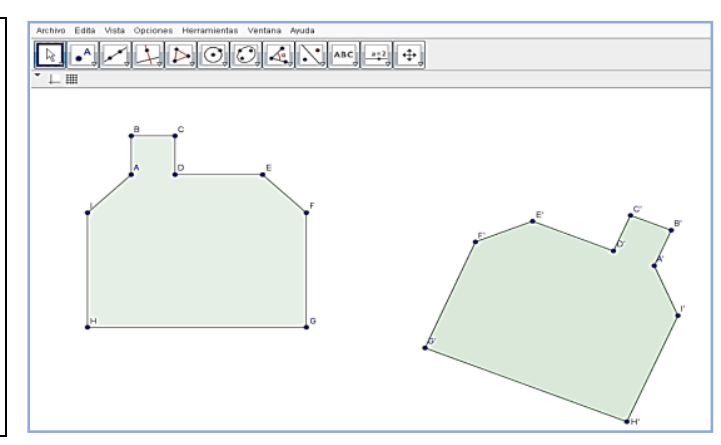

La actividad tuvo como objetivo trazar el eje de simetría no vertical, es decir, con inclinación y sin cuadrículas, dado que en la actividad $1 \mathrm{~A}$ y $1 \mathrm{~B}$ se trabajó con el eje de simetría vertical. Los estudiantes debían elegir la herramienta punto medio y traza los puntos medios de los segmentos AA', BB', CC', DD', EE', FF', GG', HH' e II’. Luego, traza la recta que pasa por los puntos medios marcados 
anteriormente. Finalmente, medir los ángulos que se forman entre la intersección de la recta y los segmentos trazados, observar que son ángulos rectos.

Para dar solución a la actividad, los posibles esquemas de uso de los estudiantes serían: recta, segmentos, punto medio, ángulos, perpendicularidad. Y el posible esquema de acción instrumentada serían eje de simetría como mediatriz de los segmentos AA', BB', CC', DD', EE', FF', GG', HH' e II'.

Una de las estudiantes llamada Marcia trazó los puntos medios de AA', BB', CC', DD', EE', FF', GG', HH' e II' y luego utilizando la herramienta recta que pasa por dos puntos, trazó la recta que contiene a todos los puntos medios. También haciendo uso de la herramienta recta que pasa por dos puntos, trazó las rectas que pasan por los puntos A y A' así como también la recta que pasa por B y B'. Posteriormente, medió los ángulos formados en la intersección de la recta $\mathrm{AA}$ ', BB' con la recta que contiene los puntos medios, tal como se muestra en la Figura 9.

\section{Figura 9}

\section{Solución de Marcia en la actividad 1C con GeoGebra (Garcia-Cuéllar, 2014)}

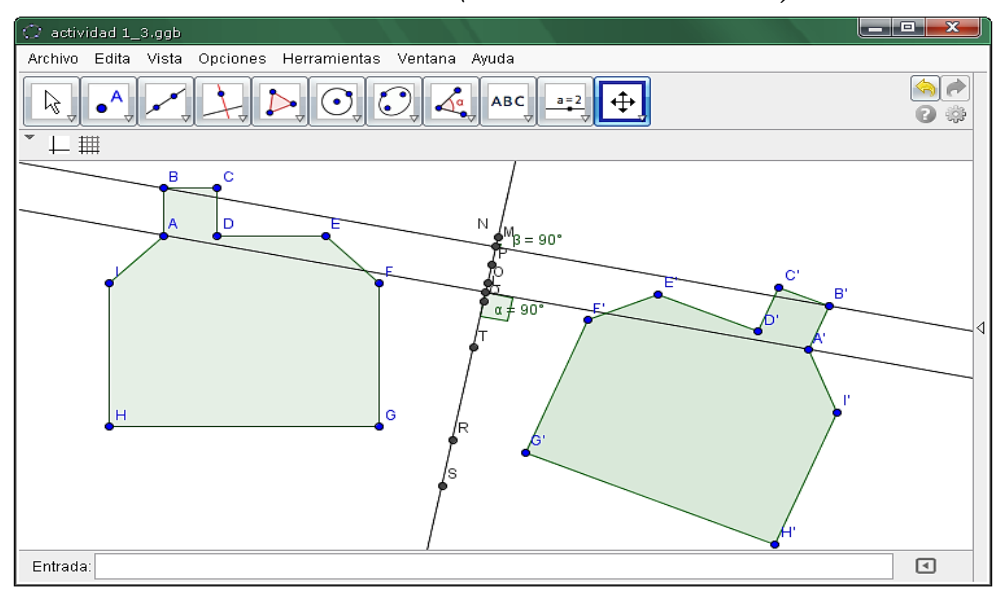

Marcía logró identificar que la recta que trazó contiene a todos los puntos medios de ambos polígonos como lo menciona en la Figura 10 (a). Asimismo, logró darse cuenta que en la intersección de los segmentos con la recta se forman ángulos rectos, tal como lo menciona en la Figura 10 (b), que muestra un recorte de su ficha de trabajo.

\section{Figura 10}

Solución de Marcia en la actividad 1C (Garcia-Cuéllar, 2014)

Abre el archivo actividadl__.ggb.Utilizando la herramienta punto medio, traza los puntos
medios de los segmentos AA', BB', CC', DD', EE', FF', GG', HH' e II'. Con la
herramienta recta, traza la recta que pasa por los puntos medios marcados anteriormente.
Anota tus observaciones.
rodos los puxtos se unen a troves de una recta.

(a)

Traza el segmento $\mathrm{AA}^{\prime}$ y mide un ángulo que se forma entre la intersección del segmento
y la recta. ¿Cuắnto mide el ángulo? $90^{\circ}$
un ángulo que se forma entre la intersección del segmento y la recta. ¿Cuánto mide el
ángulo? ¿qué puedes concluir con respectos a
los ángulos que se forman entre la intersección de la recta y los segmentos trazados?
justifica. que nunca varía su nedi da y a traves de vna recta re
pue de originar ángulos.

(b) 
Marcia logró los objetivos previstos para esta actividad. Las acciones de Marcia nos dan indicios de sus esquemas de uso como: ángulo, recta, punto medio y mediatriz. Por lo anterior, podríamos decir que generó el esquema de acción instrumentada eje de simetría como mediatriz. Dado el tiempo para resolver individualmente, la docente intervino e hizo una discusión con uso del proyector de pantalla para compartir esquemas de acción instrumentada y lograr generar esquema de acción colectiva instrumentada.

\section{ACTIVIDAD 2 A}

A seguir se presenta el enunciado y el análisis didáctico de la actividad 2A.

\section{Figura 11}

Enunciado de la Actividad 2A (Garcia-Cuéllar, 2014)

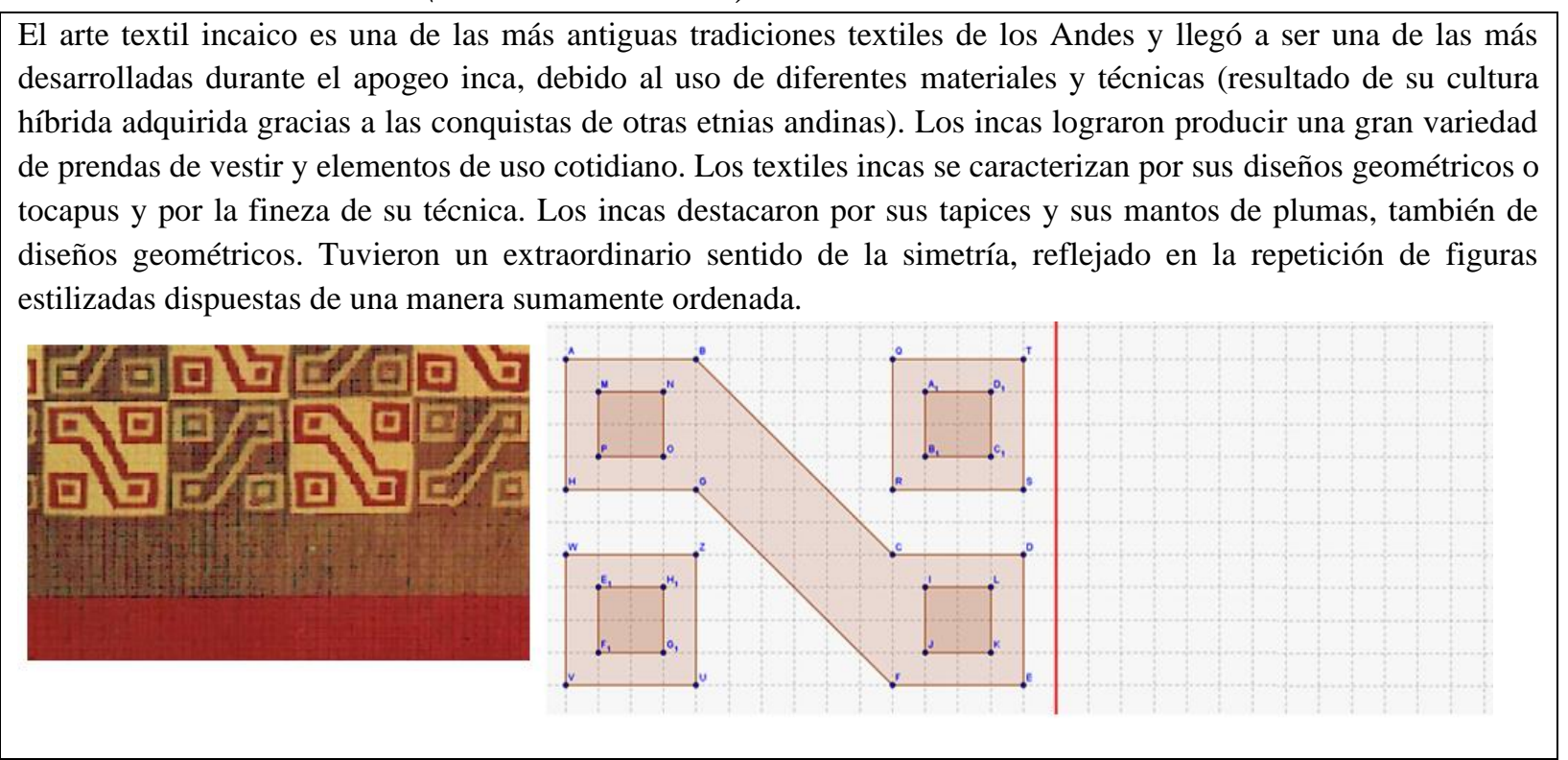

Esta actividad tuvo como objetivo realizar el simétrico de un diseño y verificar que la figura y su simétrico son equidistantes. Los estudiantes deben realizar el simétrico de cada punto de la figura manteniendo la equidistancia al eje de simetría. Luego trazar el simétrico del diseño con el uso de una regla. Esta actividad esta propuesta para realizarse con lápiz y papel, y contiene cuadrículas para que los estudiantes puedan mantener la equidistancia de cada uno de los puntos del diseño y su simétrico con respecto al eje de simetría. Los posibles esquemas de uso son simetría axial, eje de simetría, medida de segmentos, equidistancia. 


\section{Figura 12}

Enunciado de la Actividad 2A (Garcia-Cuéllar, 2014)

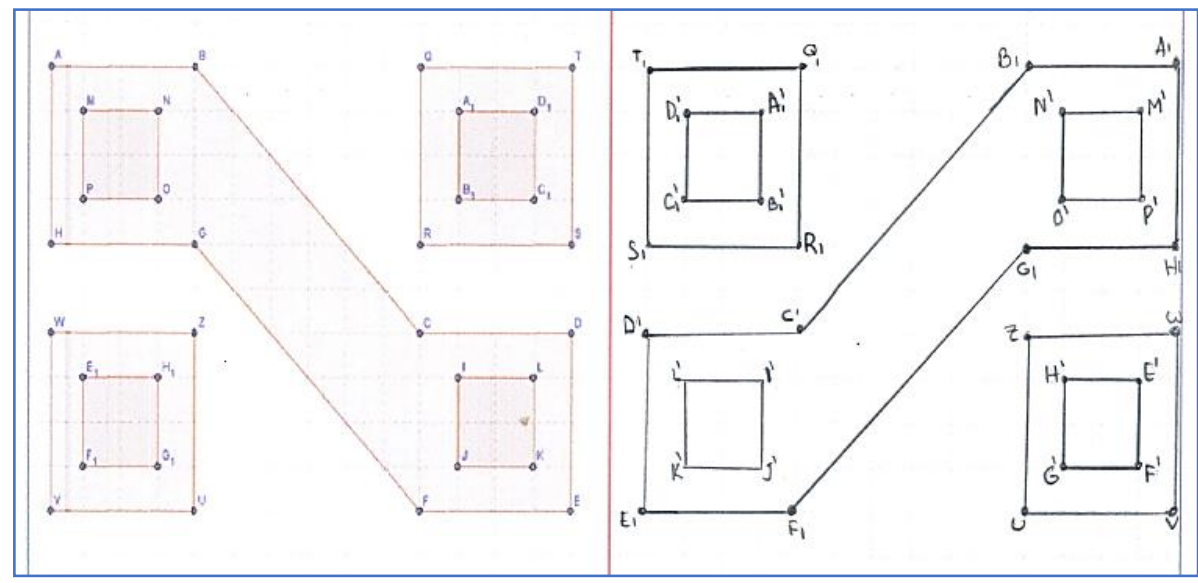

Marcía logra realizar el simétrico del diseño dado como muestra la Figura 12. Respetó la orientación del simétrico y ubicó correctamente cada uno sus puntos simétricos. Esto nos da indicios que Marcia esta instrumentada con la noción de simetría ya que lo aplica en situaciones dadas haciendo uso de GeoGebra o a lápiz y papel. Dado el tiempo para resolver individualmente, la docente intervine y realiza una discusión con uso del proyector de pantalla para compartir esquemas de acción instrumentada y lograr generar esquema de acción colectiva instrumentada.

\section{ACTIVIDAD $2 \mathrm{C}$}

A seguir se presenta el enunciado y el análisis didáctico de la actividad 2C.

\section{Figura 13}

Enunciado de la Actividad 2C (Garcia-Cuéllar, 2014)

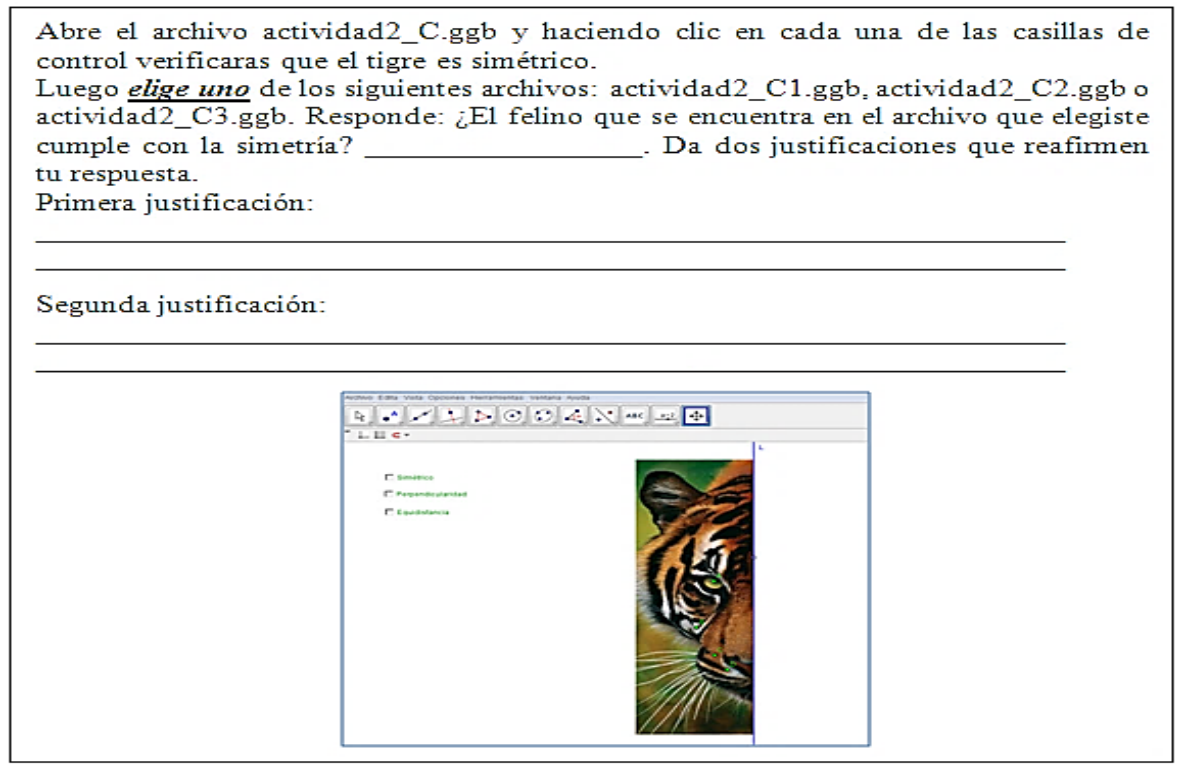


En esta actividad se presentó a los estudiantes un archivo de GeoGebra llamado Actividad2_C.ggb, donde se muestra la simetría en la imagen de un felino. Conforme iban haciendo clic en cada una de las tres casillas de control en GeoGebra (se puede ver en el link: https://www.geogebra.org/m/k3rqcerq) observan que se cumple las propiedades de la simetría. Luego, los estudiantes tenían que elegir entre tres archivos GeoGebra denominados Actividad2_C1.ggb, Actividad2_C2.ggb o Actividad2_C3.ggb. En los cuales tenían que justificar con propiedades de la simetría, si la imagen que se encontraba en el archivo elegido por ellos era simétrica o no. Los posibles esquemas de uso que pueden movilizar son la noción de eje de simetría, equidistancia, perpendicularidad.

En la Figura 14, podemos observar que los felinos de los archivos actividad2_C1.ggb y actividad2_C2.ggb, no son simétricos porque no cumple con la propiedad de la equidistancia, la perpendicularidad del eje de simetría con el segmento que une un punto de la figura inicial con su simétrico. Por el contrario, el felino que se encuentra en el archivo actividad2_C3.ggb, sí cumple con las propiedades y por ende es simétrico.

\section{Figura 14}

Solución de la actividad 2C con GeoGebra (Garcia-Cuéllar, 2014)

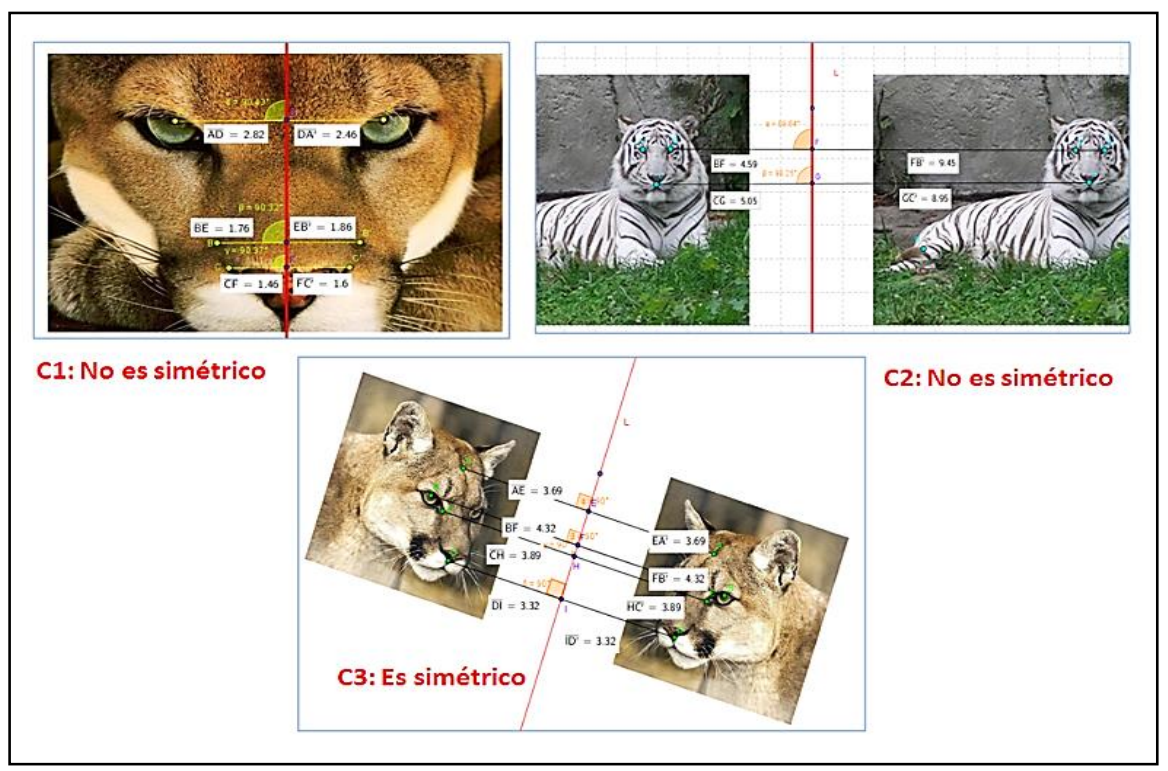

Como se pude observar en la Figura 15 (a). Marcia escogió el archivo actividad2_C1.ggb y logró reconocer que el Felino no cumplía con la simetría. Para determinarlo, utilizó las herramientas de GeoGebra que le permitieron medir ángulos, segmentos, trazar puntos de intersección. La estudiante manifestó, Figura 15 (b), que no se cumplía con la simetría porque los ángulos formados entre la intersección de los segmentos que trazó y el supuesto eje de simetría no eran perpendiculares, es decir, no median $90^{\circ}$. También que los puntos de la figura no son equidistantes con el eje de simetría. 


\section{Figura 15}

Procedimiento y respuestas de Marcia en la actividad 2C usando GeoGebra (Garcia-Cuéllar, 2014)

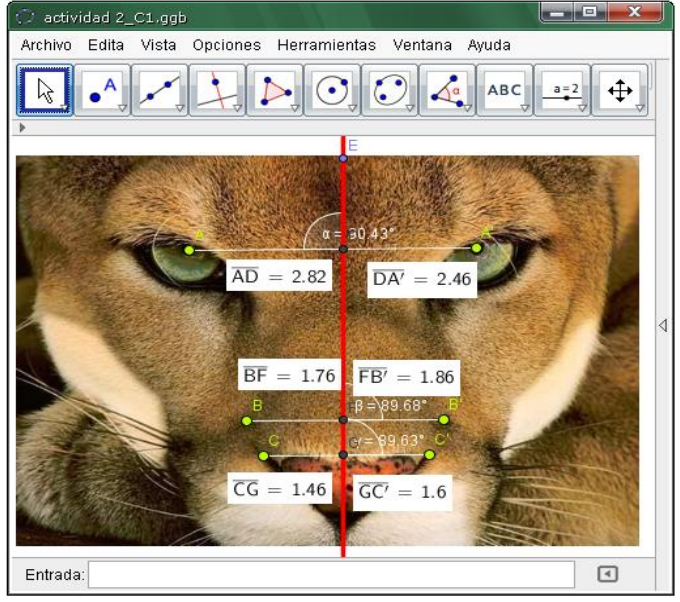

(a)

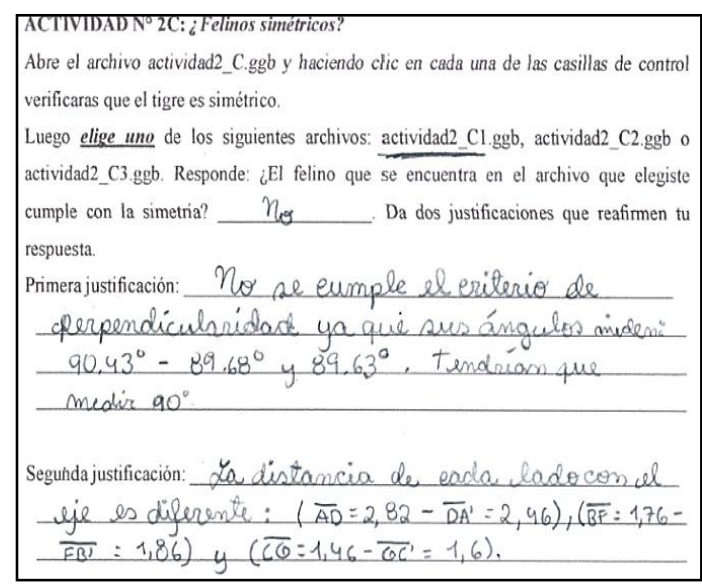

(b)

Por las acciones, antes mencionadas, y por la movilización de conceptos como eje de simetría, equidistancia y perpendicularidad, usadas para el desarrollo de esta actividad, podemos darnos cuenta que Marcia ha logrado Génesis Instrumental de la noción de simetría axial y que puede aplicar sus propiedades.

\section{FASE DESEMPEÑO DIDÁCTICO}

En cuanto a la última fase de desempeño didáctico, en donde se revisan las decisiones $A d$ hoc que toma el docente al momento de llevar a cabo la configuración didáctica, por medio del modo de ejecución, surgieron: (i) movilización de estudiantes a otras computadoras pues su computadora se reiniciaba por si sola (ii) algunas computadoras no tenían acceso a la red interna, por tanto los archivos realizados por los estudiantes fueron grabados en una memoria externa y luego grabados a la computadora de la docente. (ii) Algunos estudiantes al realizar el simétrico de una figura confundían la simetría con la traslación como se muestra en la Figura 16a, para ello, la docente indicó que realizaran la figura en GeoGebra y observaran, esto con el fin de que los estudiantes identificaran el error y deduzcan por sí solos cómo sería el simétrico de la figura y qué propiedades debería cumplir, lo cual ayudó a realizar el simétrico de la figura correctamente, Figura 16b. 


\section{Figura 16}

Respuesta de un estudiante a la actividad (datos de la investigación, Garcia-Cuéllar, 2014)

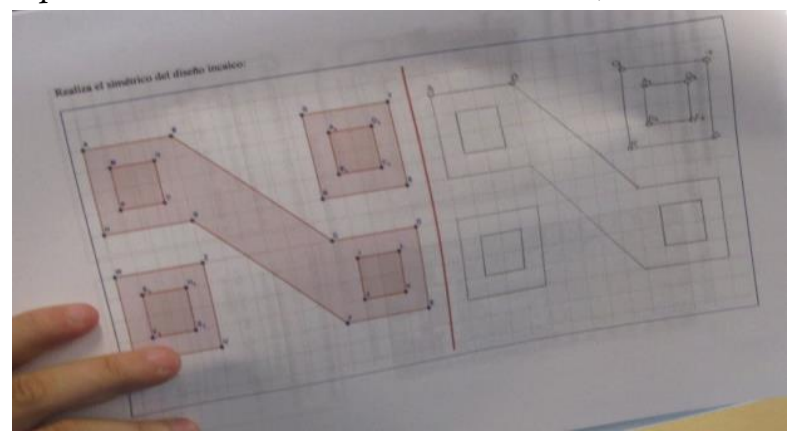

(a)

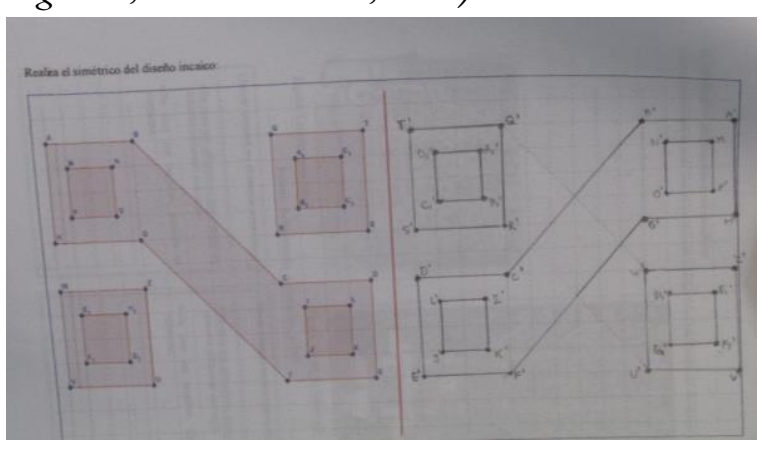

(b)

\section{CONSIDERACIONES FINALES}

A partir de la puesta en marcha de las fases de la Orquestación Instrumental para la simetría axial podemos indicar que:

En la configuración didáctica, se propusieron situaciones en donde se trabaja con lápiz y papel y otras donde se utiliza al GeoGebra lo cual nos da indicios que los artefactos tecnológicos conducen a organizar la enseñanza de maneras diferentes.

En el modo de exploración, observamos tres tipos de mediación, en el sentido de relaciones, utilizados por el docente al poner en ejecución la configuración didáctica. Estas fueron las pedagógicas, organizacional y didáctica.

En cuanto al desempeño didáctico, pusimos énfasis al seguimiento del estudiante durante la solución de las actividades; las decisiones $A d$ boc que toma la docente al momento ejecutar la configuración didáctica ayuda a reajustarlo, lo cual podemos indicar que ayuda a reconfigurar los aspectos tomados en cuenta en esa fase.

GeoGebra permitió a los estudiantes conjeturar, deducir y comprobar sus ideas para generar en un primer momento esquemas de acción instrumentada y posteriormente de una discusión entre todos se generan esquemas de acción colectiva instrumentada.

\section{ACLARATORIAS}

La autora no tiene conflictos de interés que declarar. El artículo ha sido financiado con recursos propios de la autora.

\section{REFERENCIAS}

Bellemain, F. B., \& Trouche, L. (2016). Compreender o trabalbo do professor com os recursos de seu ensino, um questionamento didático e informático [comunicación en congreso]. I Simpósio 
Latino-Americano de Didática da Matemática, Bonito, Brasil. https://hal.archivesouvertes.fr/hal-01560233

Bogdan, R., \& Biklen, S. K. (2007). Qualitative research for education. An introduction to theory and methods. 5 a edición. Pearson.

Couto, R. M. L. S. (2015). Mediaçôes didáticas da tutoria online da geometria analítica: uma análise à luz da orquestração instrumental e das representaçóes semióticas [tesis de maestría, Universidade Federal de Pernambuco]. ATTENA: Repositorio Digital de la UFPE. https://repositorio.ufpe.br/handle/123456789/18808

Drijvers, P. (2012). Teachers transforming resources into orchestrations. En G. Gueudet, B. Pepin, \& L. Trouche (Eds.), From text to "lived" resources; mathematics curriculum materials and teacher development (pp. 265-281). Springer. https://doi.org/10.1007/978-94-007-1966-8 14

Ferreira, P. (2005). Isometrias: análise de documentos curriculares e uma proposta de situaçoes de aprendizagem para o ensino médio [tesis de maestría, Pontifícia Universidade Católica de São Paulo]. Repositorio Institucional de la PUCSP. https://repositorio.pucsp.br/jspui/handle/handle/11509

Gallegos, D., \& Peña, A. (2012). Las TIC en geometría, una nueva forma de enseñar. Ediciones de la U.

García-Cuéllar, D. (2014). Simetría axial mediado por el GeoGebra: un estudio con alumnos de primer grado de educación secundaria [tesis de maestría, Pontificia Universidad Católica del Perú]. Repositorio Institucional de la PUCP. http://hdl.handle.net/20.500.12404/5651

García-Cuéllar, D., \& Salazar, J. V. F. (2019). Estudio de la génesis instrumental del artefacto simbólico simetría axial. Tangram - Revista de Educação Matemática, 2(3), 28-48. https://doi.org/10.30612/tangram.v2i3.9068

Jaime, A. (1993). Aportes a la interpretación y aplicación del modelo Van Hiele: la enseñanza de las isometrias del plano. La evaluación del nivel de razonamiento [tesis de doctorado, Universidad de Valencia]. Repositorio Institucional de la Universidad de Valencia. https://roderic.uv.es/handle/10550/37994

Ledermand, L. (1996). La partícula divina: si el universo es la respuesta, ¿cuál es la pregunta? Grijalbo Mondadori, S.A.

Lima, I. (2006). De la modélisation de connaissances des élèves aux décisions didactiques des professeurs: étude didactique dans le cas de la symétrie orthogonale [tesis de doctorado, Université Joseph Fourier - Grenoble I]. Hal Theses. https://tel.archives-ouvertes.fr/tel-00208015/ 
Perú, Ministerio de Educación (2007). Proyecto Educativo Nacional al 2021-PEN.

http://www.minedu.gob.pe/DeInteres/xtras/PEN-2021.pdf

Perú, Ministerio de Educación (2011). Orientaciones para el trabajo pedagógico 2011 del área de matemática. https://es.scribd.com/doc/75292395/OTP-matematica-2011

Perú, Ministerio de Educación (2016). Resultados de la evaluación censal de estudiantes 2016. http://umc.minedu.gob.pe/resultadosece2016/

Rabardel, P. (1995). Les hommes et les technologies: aproche cognitive des instrumentns contemporains. Armand colin.

Rabardel, P. (2011). Los hombres y las tecnologias: visión cognitiva de los instrumentos contemporáneos. (M. Acosta, trad.). Universidad Industrial de Santander.

Santillana. (2017). Matemática 1. Editorial Santillana.

Şay, R., \& Akkoç, H. (2015). Beyond orchestration: norm perspective in technology integration. En K. Krainer, \& N. Vondrová (Eds.), CERME 9 - Proceedings of the Ninth Congress of the European Society for Research in Mathematics Education (pp. 2709-2715). Charles University. https://hal.archives-ouvertes.fr/hal-01289469

Trouche, L. (2004). Managing the complexity of human/machine interactions in computerized learning environments: guiding students' command process through instrumental orchestrations. International Journal of Computers for Mathematical Learning, 9, 281-307. https://doi.org/10.1007/s10758-004-3468-5

Trouche, L., \& Drijvers, P. (2014). Webbing and orchestration. Two interrelated views on digital tools in mathematics education. Teaching Mathematics and its Applications, 1-17. https://doi.org/10.1093/teamat/hru014

Ulian, C. (2008). Possibilidades da aprendizagem de transformaçôes geométricas com o uso do CabriGeomètre [tesis de maestría, Pontificia Universidad Católica de São Paulo]. Repositorio Institucional de la PUCSP. https://repositorio.pucsp.br/jspui/handle/handle/11331

Vergnaud, G. (1996). A teoria dos campos conceptuais. En J. Brun (Ed.), Didáctica das matemáticas (pp. 155-189). Horizontes pedagógicos. 


\section{Cómo citar este artículo:}

García-Cuéllar, D. J. (2021). Una orquestación instrumental de la simetría axial. Revista Venezolana de Investigación en Educación Matemática (REVIEM), 1(2), e202102.

https://doi.org/10.54541/reviem.v1i2.11 
Copyright @ 2021. Daysi Julissa García-Cuéllar. Esta obra está protegida por una licencia Creative Commons 4.0. International (CC BY 4.0).

Usted es libre para Compartir — copiar y redistribuir el material en cualquier medio o formato - y Adaptar el documento -remezclar, transformar y crear a partir del material — para cualquier propósito, incluso para fines comerciales, siempre que cumpla la condición de:

Atribución: Usted debe dar crédito a la obra original de manera adecuada, proporcionar un enlace a la licencia, e indicar si se han realizado cambios. Puede hacerlo en cualquier forma razonable, pero no de forma tal que sugiera que tiene el apoyo del licenciante o lo recibe por el uso que hace de la obra.

$\underline{\text { Resumen de licencia }- \text { Texto completo de la licencia }}$ 